\title{
Honorarzuwachs 2010 unzureichend
}

Am 2. September 2009 hat der Erweiterte Bewertungsausschuss beschlossen, dass der bundesweit einheitliche Punktwert (Orientierungswert) für das Jahr 2010 von bisher 3,5001 Cent auf 3,5048 Cent angehoben wird. Dies entspricht einer Steigerung in Höhe von 0,134\%. Die Entscheidung der Schiedsstelle war nötig, da für diese Anpassung des Orientierungswertes für das Jahr 2010 die Kassenärztliche Bundesvereinigung (KBV) und der GKV-Spitzenverband diametral entgegengesetzte Anträge gestellt hatten. Die KBV hatte entsprechend der auf den Zeitraum zwischen den Jahren 2008 und 2010 hochgerechneten Erhöhungen der Investitions- und Betriebskosten der Praxen in Höhe von 5,3\% eine Anhebung des Orientierungswertes für 2010 in dieser Größenordnung gefordert. Die Kassenseite wollte wegen der Ausschöpfung von Wirtschaftlichkeitsreserven eine Absenkung um 6,2\%. Der Schlichter ist beiden Anträgen nicht nachgekommen, sondern hat nur die Fehlschätzungen, die der Festlegung des Orientierungswertes 2009 zugrunde lagen, aufgeschlagen. Das grundsätzliche Thema soll aber im Februar und Mai 2010 nochmals aufgegriffen werden. Dann beschäftigt sich der Bewertungsausschuss mit der Überprüfung der Kostenkalkulation des EBM und der daraus ggf. resultierenden Notwendigkeit der Angleichung von Orientierungswert und Kalkulationspunktwert des EBM in Höhe von 5,11 Cent.
Praxistipp: Nach vorläufigen Berechnungen der KBV führt der Beschluss zu einer Anhebung des Finanzvolumens 2010, das insbesondere für Leistungen im Regelleistungsvolumen zur Verfügung steht, um rund 458 Mio. Euro oder $2 \%$. In der Gesamtbetrachtung aller Leistungen der morbiditätsbedingten Gesamtvergütung zuzüglich der extrabudgetär finanzierten Leistungen und Kostenpauschalen ist von einem Zuwachs von rund 1,168 Mrd. Euro auszugehen. Dies entspricht einer Steigerung um 3,8\%. Der Anstieg des Vergütungsvolumens für außerhalb der morbiditätsbedingten Gesamtvergütung finanzierte Leistungen im Vergleich zum Volumen der morbiditätsbedingten Gesamtvergütung ergibt sich einerseits aus der Herausnahme der Dialyse-Sachkosten und andererseits aus dem nach den bisherigen Erfahrungen veranschlagten Anstieg des Ausgabenvolumens in diesem Bereich in Höhe von $8 \%$. So gesehen kann der Honorarzuwachs dort auch höher oder niedriger ausfallen. Wesentliche Elemente im extrabudgetären Bereich sind dabei weiterhin die Präventionsleistungen und die Strukturverträge, z.B. zur diabetologischen Versorgung. Da diese Leistungen aber nur von wenigen niedergelassenen Ärzten erbracht werden können, bleibt es wohl auch 2010 bei einer unzureichenden finanziellen Ausstattung der Regelleistungsvolumina und Benachteiligung der Ärzte, die ihr Leistungsspektrum v. a. dort haben.

\section{Bei Leistungen zur Unzeit genau rechnen!}

Der dringende Besuch eines Diabetikers in beschützenden Wohnheimen, Einrichtungen bzw. Pflegeoder Altenheimen mit Pflegepersonal wegen der Erkrankung kann nach Nr. 01415 EBM berechnet werden, wenn er noch am Tag der Bestellung ausgeführt wird. Diese Leistung ist außerhalb der RLV und damit praktisch extrabudgetär berechnungsfähig und mit 1545 Punkten bei einem garantierten Punktwert von 3,5001 Cent bewertet. Jeder gesondert für einen solchen Patienten im Pflegeheim bestellte Besuch ist damit nach dieser Leistung berechnungsfähig. Wird der Besuch nach 19 Uhr oder nach 22 Uhr angefordert, kann zusätzlich die Leistung nach Nr. 01100 EBM (555 P) bzw. 01101 EBM (885 P) berechnet werden. Auch diese Leistungen werden außerhalb des RLV und zum genannten festen Punktwert vergütet.

Praxistipp: Bei der geschilderten Konstellation ist klar, dass diese Art der Abrechnung nicht nur korrekt, sondern auch optimal ist. Anders sieht es bei Kon- takten zur Unzeit nach den Nrn. 01100,01101, 01411, 01412 oder 01415 EBM im Hinblick auf die Kombination mit einer der drei Versichertenpauschalen aus. Zur Verfügung stehen die Nrn. 03110-12, 03120-22 und die Versichertenpauschale nach Nr.03130 EBM. Die Versichertenpauschale nach Nr. 03130 EBM ist die ungünstigste Wahl, denn sie ist nur mit 480 Punkten bewertet. Sie muss aber angesetzt werden, wenn es im Behandlungsfall einmalig zu einem Unzeitkontakt mit dem Patienten kommt. Findet allerdings noch ein weiterer Kontakt im Behandlungsfall statt, wird die Leistung nach Nr. 03130 EBM durch die höher bewerteten Versichertenpauschalen nach den Nummern 03110 bis 03112 bzw. 03120 bis 03122 EBM ersetzt.

Da man davon ausgehen muss, dass ein Arzt im Normalfall aus der Sorgfaltspflicht heraus einen Patienten, den er notfallmäßig tags zuvor versorgt hat, nochmals zu einer Kontrolluntersuchung einbestellt, dürfte der Einsatz der Leistung nach Nr.03130 EBM extrem selten sein.

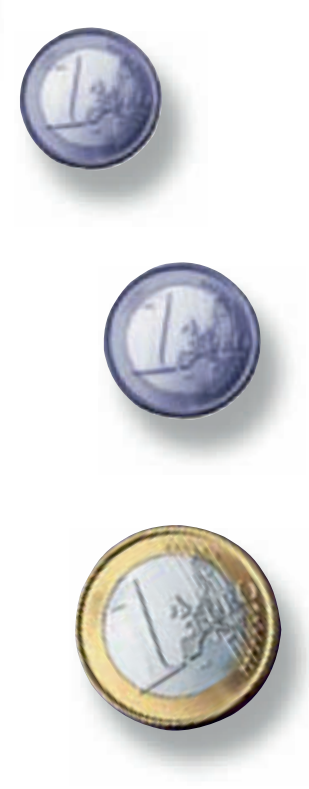

\begin{tabular}{l|l|}
\multicolumn{2}{l}{$\begin{array}{l}\text { Zuordnung } \\
\text { Punktwerte }\end{array}$} \\
\hline EBM & Punkte \\
\hline 03110 & 1020 \\
03111 & 900 \\
\hline 03112 & 1000 \\
\hline 03120 & 500 \\
\hline 03121 & 450 \\
\hline 03122 & 535 \\
\hline 03130 & 480 \\
\hline
\end{tabular}

